Case Report

\title{
Pulmonary Limited MPO-ANCA Microscopic Polyangiitis and Idiopathic Lung Fibrosis in a Patient with a Diagnosis of IgA Nephropathy
}

\author{
Alwin Tilanus, ${ }^{1}$ Patricia Van der Niepen, ${ }^{2}$ Caroline Geers, ${ }^{3}$ and Karl Martin Wissing ${ }^{2}$ \\ ${ }^{1}$ Departamento de Medicina Interna/Infectologia, Hospital General de Medellin Luz Castro de Gutiérrez, \\ Carrera 48 \# 32-102, Medellin, Colombia \\ ${ }^{2}$ Departement Interne Geneeskunde/Nefrologie, Universitair Ziekenhuis Brussel, Laarbeeklaan 101, 1090 Brussels, Belgium \\ ${ }^{3}$ Departement Anatomo-Pathologie, Universitair Ziekenhuis Brussel, Laarbeeklaan 101, 1090 Brussels, Belgium
}

Correspondence should be addressed to Alwin Tilanus; alwintilanus@hotmail.com

Received 31 March 2015; Revised 22 June 2015; Accepted 28 June 2015

Academic Editor: Mahzuz Karim

Copyright (C) 2015 Alwin Tilanus et al. This is an open access article distributed under the Creative Commons Attribution License, which permits unrestricted use, distribution, and reproduction in any medium, provided the original work is properly cited.

\begin{abstract}
We present a case of a male patient with chronic renal insufficiency, due to crescentic glomerulonephritis with IgA deposits, who successively developed (idiopathic) thrombocytopenic purpura (ITP) and MPO-ANCA microscopic polyangiitis (MPA) with pulmonary fibrosis. The patient presented with cough, weight loss, and dyspnea on exertion. CT imaging and pulmonary function tests were compatible with interstitial pneumonitis with pulmonary fibrosis. Laboratory results showed high MPO-ANCA titers; the urinary sediment was bland. The patient was treated successfully with cyclophosphamide and methyl-prednisolone. This unique case illustrates the diagnostic and therapeutic challenges of an unusual presentation of microscopic polyangiitis presenting first as isolated kidney disease with recurrence in the form of pneumonitis without renal involvement, in association with renal IgA deposits and ITP as coexisting autoimmune conditions.
\end{abstract}

\section{Introduction}

The association between pulmonary fibrosis $(\mathrm{PF})$ and Myeloperoxidase Anti-Neutrophil Cytoplasmic Antibodies (MPOANCAs) positivity has been reported in several small retrospective case-series, but the pathologic mechanism remains unclear [1]. In published case series [2-8] most patients were men with an average age of about 70 years, presenting typically with dry cough and dyspnea.

In a large proportion of cases, the diagnosis of MPOANCA vasculitis was preceded months to years by the diagnosis of PF or signs of interstitial pneumonia. During followup prognosis is unfavorable due to respiratory failure [2-8].

MPO-ANCAs have been reported in $7 \%$ to $33 \%$ of patients with interstitial pneumonia and idiopathic pulmonary fibrosis. Along the same line pulmonary fibrosis has been detected in one-third of a cohort of patients with pANCA-MPO $[5,7,9]$.
Studies of patients with ANCA-positive and ANCA-negative PF demonstrated no significant differences between these clinical entities regarding symptoms, pulmonary function tests, and CT scanning $[1,5]$. In most patients, more than one organ is affected, most frequently the kidney.

Here we report a unique case of MPO-ANCA microscopic polyangiitis affecting primarily the lung in a patient with a history of MPO-ANCA microscopic polyangiitis with renal-limited vasculitis and coexisting IgA deposits as well as idiopathic thrombocytopenic purpura (ITP).

\section{Case Report}

A 71-year-old man was admitted in June 2012 because of progressive dyspnea on exertion, dry cough, progressive weight loss of approximately $20 \mathrm{~kg}$ in 3 months, and edema of the lower extremities.

He had a complex medical history of autoimmune diseases including chronic kidney disease from a biopsy-proven 


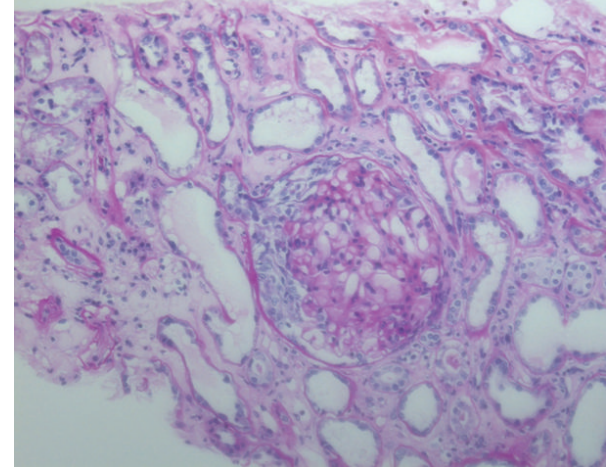

FIgURE 1: Periodic acid-Schiff stained histologic preparation with an overview of the renal parenchyma. The glomerulus shows a cellular crescent. The glomerular tuft shows mesangial hypercellularity. Tubular cells are damaged with regenerative features (PAS, $\times 200$ ).

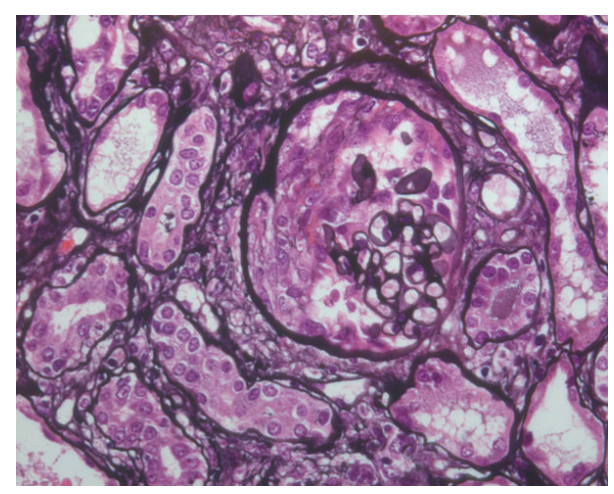

FIGURE 2: The methenamine silver stain shows fibrinoid necrosis with early crescent formation in the urinary space (Jones methenamine silver, $\times 400$ ).

crescentic glomerulonephritis with MPO-ANCA and coexistent glomerular IgA deposits in 2007 and idiopathic thrombocytopenic purpura (ITP) in 2011. The patient had never smoked.

In December 2007 he was hospitalized because of acute renal failure with serum creatinine $>1060 \mu \mathrm{mol} /$ liter, oliguria, microscopic hematuria $(250 \mathrm{RBC} / \mu \mathrm{L})$, and proteinuria (1.3 g/day). Furthermore, he had an inflammatory syndrome (CRP $194 \mathrm{mg} / \mathrm{liter}$ ) and very high ANCA (>1/5120) and antiMPO (2337 IU/mL) titers.

Renal biopsy (2007) showed necrotizing crescentic glomerulonephritis (Figures 1 and 2) with significant IgA deposits (not shown in figure).

The patient received a diagnosis of severe IgA nephropathy with crescentic glomerulonephritis and was initially treated with high dose corticosteroids and hemodialysis. His kidney function progressively recovered and he was able to stop dialysis therapy after 3 months. Between June 2008 and 2011 serum creatinine values stabilized at values between 206 and $252 \mu \mathrm{mol} /$ liter with maintenance methyl-prednisolone of approximately $8 \mathrm{mg}$ every other day. Microscopic hematuria and proteinuria resolved and anti-MPO antibodies decreased from $2337 \mathrm{IU} / \mathrm{mL}$ to $208 \mathrm{IU} / \mathrm{mL}$ by April 2009.
In October 2011 the patient was hospitalized for epistaxis and severe thrombocytopenia $7 * 10^{3} / \mathrm{mm}^{3}$ in the absence of anemia or leucopenia. The patient had no clinical signs of recurring renal disease or vasculitis and anti-MPO ANCA had further decreased to $45 \mathrm{IU} / \mathrm{mL}$. Bone marrow examination was performed with diagnosis of idiopathic thrombocytopenia. Platelets rapidly normalized after short course of intravenous methyl-prednisolone followed by increased doses of maintenance steroids.

Interstitial pneumonia with beginning pulmonary fibrosis was diagnosed in November 2011 during evaluation for progressive dyspnea. A Broncho Alveolar Lavage (BAL) (performed under corticoid treatment) showed no abnormalities.

During the following six months his clinical condition progressively worsened in spite of treatment with high dose steroids and the patient was hospitalized several times for corticosteroid-induced diabetes, herpes zoster infection, and cellulitis of the right leg.

When admitted in June 2012 he appeared severely ill, wheelchair bound, with symmetric muscle wasting and loss of strength and resting dyspnea. He was profoundly anorectic with a loss of $27 \mathrm{~kg}$ since October 2011 and of $16 \mathrm{~kg}$ during the two month before admission. The examination of the chest revealed normal heart tones and bibasal fine lung crackles with oxygen saturation at $93 \%$. Examination of the abdomen was unremarkable. Venous insufficiency and slight pitting edema were present at both lower limbs.

Laboratory examination showed a normocytic anemia $\mathrm{Hb} 7.0 \mathrm{~g} / \mathrm{dL}$, normal leukocyte count, an elevated C-reactive protein (CRP $246 \mathrm{mg} /$ liter), and erythrocyte sedimentation rate $($ ESR $99 \mathrm{~mm} / \mathrm{h})$. Serum creatinine was stable at $203 \mu \mathrm{mol} /$ liter; the urinary sediment showed no red or white blood cells and no casts; no proteinuria was detected. The patient had normal levels of eosinophils.

As compared to December 2011 and in spite of high dose steroids, p-ANCA titers had increased from 1/80 to 1/640 U/ $\mathrm{mL}$ and MPO antibodies were above the upper limit of the titration curve of the test.

During the same period, spirometry showed a remarkable worsening of the restrictive pattern with forced expiratory volume in 1 second (FEV1) decreasing from $1.99 \mathrm{~L}$ to $0.71 \mathrm{~L}$ and diminishing carbon monoxide (CO) diffusion capacity of the lung.

CT scan of the thorax was classified as usual interstitial pneumonia (UIP) with reticular infiltrates and honeycombing predominantly in the subpleural areas of the lower lobes, without possibility to differentiate between idiopathic pulmonary fibrosis and fibrosis secondary to vasculitis on radiological grounds (Figure 3). Giant vessel vasculitis was excluded by PET-CT scan (though performed under corticosteroid treatment). A biopsy of the nasal mucosa showed nonspecific inflammation without signs of vasculitis or granulomatous lesions. There were no signs of active renal disease with a bland urinary sediment and absence of proteinuria as well as a stable but decreased renal function (CKD stage 3B).

Because of inflammation in the blood and a sputum culture positive for Moraxella/Proteus, the patient was treated with amoxicillin/clavulanic acid. This treatment was complicated by the development of an extensive erythematous 
TABLE 1: Change of p-ANCA, MPO, spirometry, and CT scanning characteristics in the period from December 2011 to October 2012.

\begin{tabular}{|c|c|c|c|}
\hline & \multicolumn{3}{|c|}{ Period } \\
\hline & December 2011 & June 2012 & $\begin{array}{c}\text { October } 2012 \text { (after } 5 \\
\text { cycles of } \\
\text { cyclophosphamide) }\end{array}$ \\
\hline p-ANCA & $1 / 180$ & $1 / 640$ & $1 / 320$ \\
\hline MPO (IU/L) & 45 & $>134^{1}$ & 44 \\
\hline \multicolumn{4}{|l|}{ Spirometry } \\
\hline FVC (forced vital capacity) (liters) & $2.51\left(58 \%\right.$ of $\left.\mathrm{PV}^{2}\right)$ & $0.84(20 \%$ of PV $)$ & $2.23(52 \%$ of PV) \\
\hline $\begin{array}{l}\text { Forced expiratory volume } 1 \text { second } \\
\text { (FEV1) (liters) }\end{array}$ & $\begin{array}{c}1.99 \\
(61 \% \text { of PV })\end{array}$ & $\begin{array}{c}0.71 \\
(22 \% \text { of PV })\end{array}$ & $\begin{array}{c}1.63 \\
(50 \% \text { of PV) }\end{array}$ \\
\hline FEC/FVC \% & $79(74 \%$ of PV) & $84 \%(74 \%$ of $\mathrm{PV})$ & $73 \%(74 \%$ of $\mathrm{PV})$ \\
\hline VC (vital capacity) (liters) & $2.51(56 \%$ of PV) & $0.98(22 \%$ of PV $)$ & $2.23(50 \%$ of $\mathrm{PV})$ \\
\hline TLC (total lung capacity) (liters) & $3.84(51 \%$ of PV) & Not measured & $3.96(53 \%$ of PV) \\
\hline RV (residual volume) (liters) & $1.33(49 \%$ of PV) & Not measured & $1.73(63 \%$ of PV) \\
\hline $\begin{array}{l}\text { DLCO (diffusing capacity of the lung for } \\
\text { carbon monoxide) }(\mathrm{mL} / \mathrm{mmHg} / \mathrm{min})\end{array}$ & $\begin{array}{c}9.2 \\
(32 \% \text { of PV })\end{array}$ & Not measured & $\begin{array}{c}7.6 \\
(27 \% \text { of PV })\end{array}$ \\
\hline CT scanning & $\begin{array}{l}\text { Interstitial pneumonia/idiopathic } \\
\text { lung fibrosis }\end{array}$ & $\begin{array}{l}\text { Interstitial pneumonia/idiopathic } \\
\text { lung fibrosis }\end{array}$ & $\begin{array}{c}\text { Unchanged } \\
\text { (permanent damage) }\end{array}$ \\
\hline
\end{tabular}

${ }^{1}$ Protocolled as $>134$ IU (above upper limit of test range).

${ }^{2}$ PV: Predicted Value.

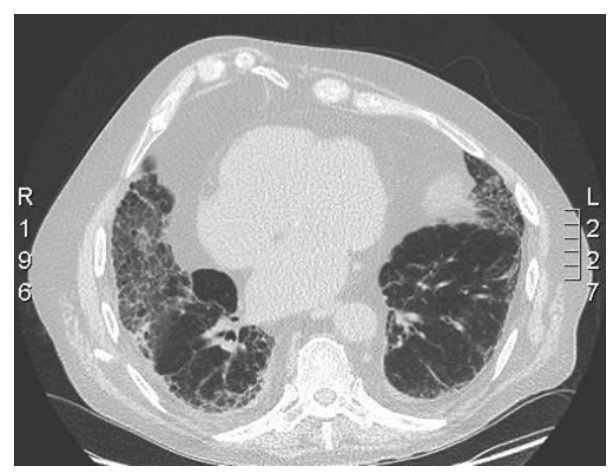

FIGURE 3: CT scan (prior to treatment) showing diffuse pulmonary fibrosis (honeycombing) and bilateral pleural fluid collection.

skin rash spreading over the entire body. Skin biopsies showed diffuse infiltrates with eosinophils and neutrophils and were protocoled as drug-induced rash. Antibiotic treatment was changed to moxifloxacine and $32 \mathrm{mg} /$ day methylprednisolone with improvement of the rash. At that time the patient was considered too ill to undergo open lung biopsy for a formal histological diagnosis of fibrosis and/or vasculitis. In the context of increasing ANCA, an inflammatory syndrome as well as anorexia and severe weight loss microscopic polyangiitis with rapidly progressive pulmonary fibrosis was considered as sufficiently likely to justify the initiation of combination therapy with intravenous cyclophosphamide and high dose oral corticosteroids according to the CYCLOPS protocol [10].

Within days after the first cycle of cyclophosphamide we observed remarkable clinical improvement with decrease in dyspnea, increased appetite, and improved gait.

After 5 cycles of IV cyclophosphamide, pANCA/MPO titers decreased from $1 / 640$ to $1 / 320$ and 134 to $44 \mathrm{U} / \mathrm{mL}$, respectively, and inflammatory parameters normalized. Furthermore, FEV1 significantly improved from 0.71 to $1.63 \mathrm{~L}$, Table 1. The CT scan showed no changes. The patient was last seen at the outpatient clinic in December 2012 with a serum creatinine of $190 \mu \mathrm{mol} / \mathrm{L}$, hemoglobin levels of $11.4 \mathrm{~g} / \mathrm{dL}$, and a CRP of $8.6 \mathrm{mg} / \mathrm{L}$. Weight increased from 81 to $91 \mathrm{~kg}$ during the 6 months after starting cyclophosphamide and the patient was again able to walk without assistance. He received a maintenance immunosuppression with azathioprine and Medrol $4 \mathrm{mg} /$ day and no longer returned for follow-up at our unit.

\section{Discussion}

The present case is characterized by both the unusual association of several autoimmune conditions and a sequential and atypical presentation of microscopic polyangiitis, with selective damage of first the kidneys and subsequently the lungs without recurring of kidney disease. The initial presentation of our patient with rapidly progressive renal failure, high titers of MPO-ANCA, and crescentic glomerulonephritis with fibrinoid necrosis was clearly suggestive of renal-limited microscopic polyangiitis. The patient received a diagnosis of aggressive IgA nephropathy at this moment because of the presence of mesangial IgA deposits. Similar to our patient, several reports have described patients with (crescentic) IgA nephropathy in the presence of positive ANCAs [11-14]. It is unclear whether IgA nephropathy precedes ANCA positivity or if mesangial inflammation due to $\operatorname{IgA}$ deposits creates an inflammatory microenvironment that triggers vasculitis. Similar to our patient the clinical presentation is in general characterized by rapid loss of renal function and requires aggressive immunosuppressive therapy combining cyclophosphamide and steroids. Although our patient ultimately entered remission and was able to stop dialysis therapy, in retrospect we have to conclude that he should have been treated for MPA at the first presentation of his disease. 
Thrombotic and thrombocytopenic purpura (TTP) has been described in rare patients with ANCA-associated vasculitis [15-17]; there has been, to the best of our knowledge, no previous report on the association between idiopathic thrombocytopenic purpura and ANCA-associated vasculitis. The relation of ITP with vasculitis in our patient is not clear because it occurred at a moment when MPO-ANCA titers were low but ITP was also rapidly followed by recurrence of vasculitis in the form of pulmonary fibrosis. Severe thrombocytopenia rapidly responded to high dose steroids and did not recur during the rest of follow-up.

Our patient developed progressive interstitial pneumonitis and pulmonary fibrosis that was first diagnosed approximately one month after the development of ITP and occurred in spite of the administration of high dose steroids for ITP and initially without increase in MPO-ANCA and other signs of MPA. The link with ANCA vasculitis was made only 6 months later when the patient had developed severe systemic disease in the form of anorexia, wasting, anemia, and an inflammatory syndrome in the context of high MPO-ANCA titers. Surprisingly, although MPA had initially presented as renal limited disease, the recurrence occurred without any signs of renal involvement. The presentation of our patient is compatible with the largest series published up to now which reported a predominance of older male patients with MPOANCA among those developing $\mathrm{PF}$ and a majority presenting with a typical UIP pattern on CT scans [8]. Homma et al. examined 31 patients diagnosed as having $\mathrm{PF}$ with positive MPO-ANCA. In 11 autopsied patients the histopathological features of the diseased lung tissues were compatible with the usual interstitial pneumonia pattern. Vasculitis in bronchial arteries and/or pulmonary arterioles was confirmed in only five patients [6]. Hervier et al. reported low diagnostic yield of 3 transbronchial biopsies which showed only fibrosis and no vasculitis [4]. On the contrary a large series of patients with open lung biopsy (25) or autopsy (2) provided a correct diagnosis of vasculitis in 21 of 27 ANCA positive patients (78\%), capillaritis being the most common lesion [18]. In the present patient we decided against transbronchial biopsy because of the low diagnostic yield and against open lung biopsy because the patient was considered too ill to undergo surgery. The treating physicians had also decided that systemic inflammation with anorexia and wasting in the context of high titers of MPO-ANCA were a justification of standard treatment of MPA irrespective of the results of an eventual open lung biopsy.

The pathologic mechanism of pulmonary fibrosis and positive MPO-ANCA remains unclear [1]. There is evidence that infection and certain drugs can stimulate ANCA-MPO/ PR3 antibody production [19]. Infection can prime and activate neutrophils by circulating inflammatory cytokines. Subsequent translocation of ANCA antigens (e.g., MPO/PR3) to the cell surface of neutrophils and expression of adhesion molecules by endothelial cells result in cell adhesion, release of reactive oxygen species, vasculitis, and endothelial apoptosis [20]. Chan et al. described a case of MPO-ANCA microscopic vasculitis following a suppurative wound infection in a cancer patient [21]. Infectious events in our patient (cellulitis right leg, pneumonia) might have triggered MPOANCA titers increase. Takato et al. described a case of an MPO-ANCA positive PF patient in which a Mycoplasma infection triggered the elevation of MPO-ANCA titers and the development of a crescentic glomerulonephritis [22].

When examining the published case series [2-8], prognosis was typically worse when PF was associated with MPOANCA positivity especially when eosinophilia was present [4]. The MPO-ANCA antibodies could lead to pulmonary capillaritis which might result in (subclinical) alveolar hemorrhage and finally fibrosis [23-26]. Nozu et al. suggested that there is some evidence that corticosteroid therapy is more effective in ANCA positive patients and that survival tends to be better when the MPO titers were low (<50 IU/L) [5]. Nevertheless, and similar to the initial clinical presentation of our patient who developed fibrosis before increasing MPO titers, the development of PF has been reported to precede increase in MPO-ANCA positivity. In about half of the patients the diagnosis of PF precedes the diagnosis of vasculitis [8]. Therefore the opposite theory that PF could result in MPO-ANCA production and be a trigger for the development or recurrence of MPA cannot be discarded $[4,7]$. The reported lack of clear correlation between MPOANCA titers and severity of PF also question the unique role of ANCA in the disease process [6].

Although in our case the lungs were the only vital organ affected, other organs might have been subsequently damaged during disease progression as previously reported by Hiromura et al. In this report, four patients with MPOANCA positive idiopathic pulmonary fibrosis subsequently developed rapidly progressive glomerulonephritis [27].

Our patient was treated according to the vasculitis guidelines (CYCLOPS protocol [10]) with rapid clinical improvement. Renal function improved and MPO-ANCA titers decreased significantly. A control spirometry after 5 cycles of cyclophosphamide showed a remarkable improvement in total lung volume and FEV1. However, a control pulmonary CT scan was unchanged, indicating that most lung tissue damage was irreversible.

Since high (e.g., $>50 \mathrm{IU} / \mathrm{mL}$ ) MPO-ANCA titers in a patient with PF carry a worse prognosis and risk of evolution to MPA, every patient with (idiopathic) PF should be screened for MPO-ANCA positivity. When positive, we propose to look for underlying infections, treat them as necessary, and start treatment with cyclophosphamide/corticosteroids, even without prior lung biopsy.

\section{Conflict of Interests}

The authors declare that they have no conflict of interests.

\section{References}

[1] H. Yamada, "ANCA: associated lung fibrosis," Seminars in Respiratory and Critical Care Medicine, vol. 32, no. 3, pp. 322-327, 2011.

[2] G. M. Eschun, S. N. Mink, and S. Sharma, "Pulmonary interstitial fibrosis as a presenting manifestation in perinuclear 
antineutrophilic cytoplasmic antibody microscopic polyangiitis," Chest, vol. 123, no. 1, pp. 297-301, 2003.

[3] G. Foulon, P. Delaval, D. Valeyre et al., "ANCA-associated lung fibrosis: analysis of 17 patients," Respiratory Medicine, vol. 102, no. 10, pp. 1392-1398, 2008.

[4] B. Hervier, C. Pagnoux, C. Agard et al., "Pulmonary fibrosis associated with ANCA-positive vasculitides. Retrospective study of 12 cases and review of the literature," Annals of the Rheumatic Diseases, vol. 68, no. 3, pp. 404-407, 2009.

[5] T. Nozu, M. Kondo, K. Suzuki, J. Tamaoki, and A. Nagai, "A comparison of the clinical features of ANCA-positive and ANCA-negative idiopathic pulmonary fibrosis patients," Respiration, vol. 77, no. 4, pp. 407-415, 2009.

[6] S. Homma, H. Matsushita, and K. Nakata, "Pulmonary fibrosis in myeloperoxidase antineutrophil cytoplasmic antibodyassociated vasculitides," Respirology, vol. 9, no. 2, pp. 190-196, 2004.

[7] G. E. Tzelepis, M. Kokosi, A. Tzioufas et al., "Prevalence and outcome of pulmonary fibrosis in microscopic polyangiitis," European Respiratory Journal, vol. 36, no. 1, pp. 116-121, 2010.

[8] C. Comarmond, B. Crestani, A. Tazi et al., "Pulmonary fibrosis in antineutrophil cytoplasmic antibodies (ANCA)-associated vasculitis: a series of 49 patients and review of the literature," Medicine, vol. 93, no. 24, pp. 340-349, 2014.

[9] A. Shiraki, M. Ando, J. Shindoh et al., "Prevalence of myeloperoxidase-anti-neutrophil cytoplasmic antibody (MPO-ANCA) in patients with interstitial pneumonia," Nihon Kokyuki Gakkai Zasshi, vol. 45, no. 12, pp. 921-926, 2007.

[10] K. De Groot, L. Harper, D. R. W. Jayne et al., "Pulse versus daily oral cyclophosphamide for induction of remission in antineutrophil cytoplasmic antibody-associated vasculitis: a randomized trial," Annals of Internal Medicine, vol. 150, no. 10, pp. 670-680, 2009.

[11] M. Haas, J. Jafri, S. M. Bartosh, S. L. Karp, S. G. Adler, and S. M. Meehan, "ANCA-associated crescentic glomerulonephritis with mesangial IgA deposits," The American Journal of Kidney Diseases, vol. 36, no. 4, pp. 709-718, 2000.

[12] S. L. Lui, K. W. Chan, P. S. Yip, T. M. Chan, K. N. Lai, and W. K. Lo, "Simultaneous occurrence of diabetic glomerulosclerosis, IgA nephropathy, crescentic glomerulonephritis, and myeloperoxidase-antineutrophil cytoplasmic antibody seropositivity in a Chinese patient," American Journal of Kidney Diseases, vol. 40, no. 4, pp. el4.1-e14.4, 2002.

[13] J. Ara, J. Bonet, R. Rodríguez, E. Mirapeix, I. Agraz, and R. Romero, "IgA nephropathy with crescentic glomerulonephritis and ANCA positive," Nefrologia, vol. 25, no. 6, pp. 712-717, 2005.

[14] C. Bantis, M. Stangou, C. Schlaugat et al., "Is presence of ANCA in crescentic IgA nephropathy a coincidence or novel clinical entity? A case series," The American Journal of Kidney Diseases, vol. 55, no. 2, pp. 259-268, 2010.

[15] V. Agrawal, C. K. Vaidya, J. Ye et al., "Concomitant thrombotic thrombocytopenic purpura and ANCA-associated vasculitis in an adolescent," Pediatric Nephrology, vol. 26, no. 8, pp. 1317-1320, 2011.

[16] H. Watanabe, W. Kitagawa, K. Suzuki et al., "Thrombotic thrombocytopenic purpura in a patient with rapidly progressive glomerulonephritis with both anti-glomerular basement membrane antibodies and myeloperoxidase anti-neutrophil cytoplasmic antibodies," Clinical and Experimental Nephrology, vol. 14, no. 6, pp. 598-601, 2010.

[17] K. Nagai, T. Kotani, T. Takeuchi et al., "Successful treatment of thrombotic thrombocytopenic purpura with repeated plasma exchange in a patient with microscopic polyangitis," Modern Rheumatology, vol. 18, no. 6, pp. 643-646, 2008.

[18] P. B. Gaudin, F. B. Askin, R. J. Falk, and J. C. Jennette, “The pathologic spectrum of pulmonary lesions in patients with antineutrophil cytoplasmic autoantibodies specific for anti-proteinase 3 and anti-myeloperoxidase," American Journal of Clinical Pathology, vol. 104, no. 1, pp. 7-16, 1995.

[19] E. Csernok, P. Lamprecht, and W. L. Gross, "Clinical and immunological features of drug-induced and infection-induced proteinase 3-antineutrophil cytoplasmic antibodies and myeloperoxidase-antineutrophil cytoplasmic antibodies and vasculitis," Current Opinion in Rheumatology, vol. 22, no. 1, pp. 43-48, 2010.

[20] C. G. M. Kallenberg, "Pathogenesis of ANCA-associated vasculitides," Annals of the Rheumatic Diseases, vol. 70, supplement 1, pp. i59-i63, 2011.

[21] B. Chan, V. d'Intini, and J. Savige, "Anti-neutrophil cytoplasmic antibody (ANCA)-associated microscopic polyangiitis following a suppurative wound infection," Nephrology Dialysis Transplantation, vol. 21, no. 10, pp. 2993-2994, 2006.

[22] H. Takato, M. Yasui, Y. Waseda, N. Sakai, T. Wada, and M. Fujimura, "A case of microscopic polyangiitis following mycoplasma infection in a patient with MPO-ANCA positive pulmonary fibrosis," Allergology International, vol. 60, no. 1, pp. 9396, 2011.

[23] A. Schnabel, M. Reuter, E. Csernok, C. Richter, and W. L. Gross, "Subclinical alveolar bleeding in pulmonary vasculitides: correlation with indices of disease activity," European Respiratory Journal, vol. 14, no. 1, pp. 118-124, 1999.

[24] J.-F. Cordier and V. Cottin, "Alveolar hemorrhage in vasculitis: primary and secondary," Seminars in Respiratory and Critical Care Medicine, vol. 32, no. 3, pp. 310-321, 2011.

[25] P. Manganelli, P. Fietta, M. Carotti, A. Pesci, and F. Salaffi, "Respiratory system involvement in systemic vasculitides," Clinical and Experimental Rheumatology, vol. 24, no. 2, pp. S48-S59, 2006.

[26] J. Birnbaum, S. Danoff, F. B. Askin, and J. H. Stone, "Microscopic polyangiitis presenting as a 'pulmonary-muscle' syndrome: is subclinical alveolar hemorrhage the mechanism of pulmonary fibrosis?" Arthritis and Rheumatism, vol. 56, no. 6, pp. 20652071, 2007.

[27] K. Hiromura, Y. Nojima, T. Kitahara et al., "Four cases of antimyeloperoxidase antibody-related rapidly progressive glomerulonephritis during the course of idiopathic pulmonary fibrosis," Clinical Nephrology, vol. 53, no. 5, pp. 384-389, 2000. 


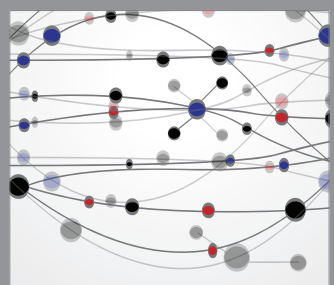

The Scientific World Journal
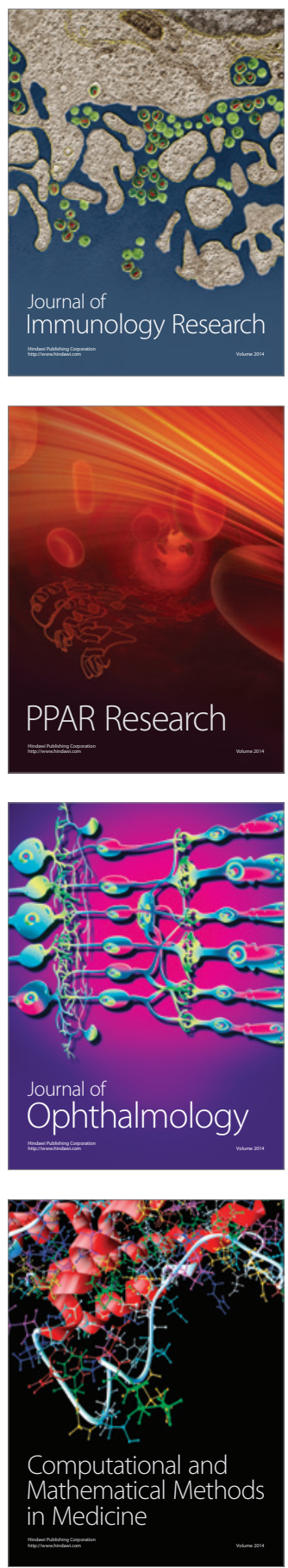

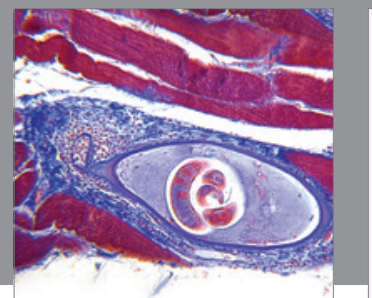

Gastroenterology

Research and Practice
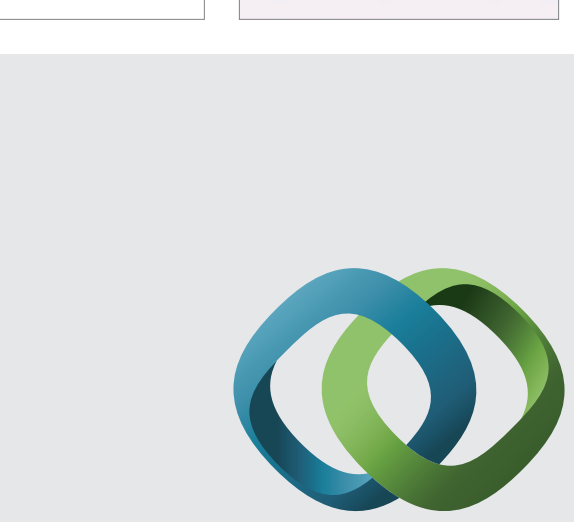

\section{Hindawi}

Submit your manuscripts at

http://www.hindawi.com
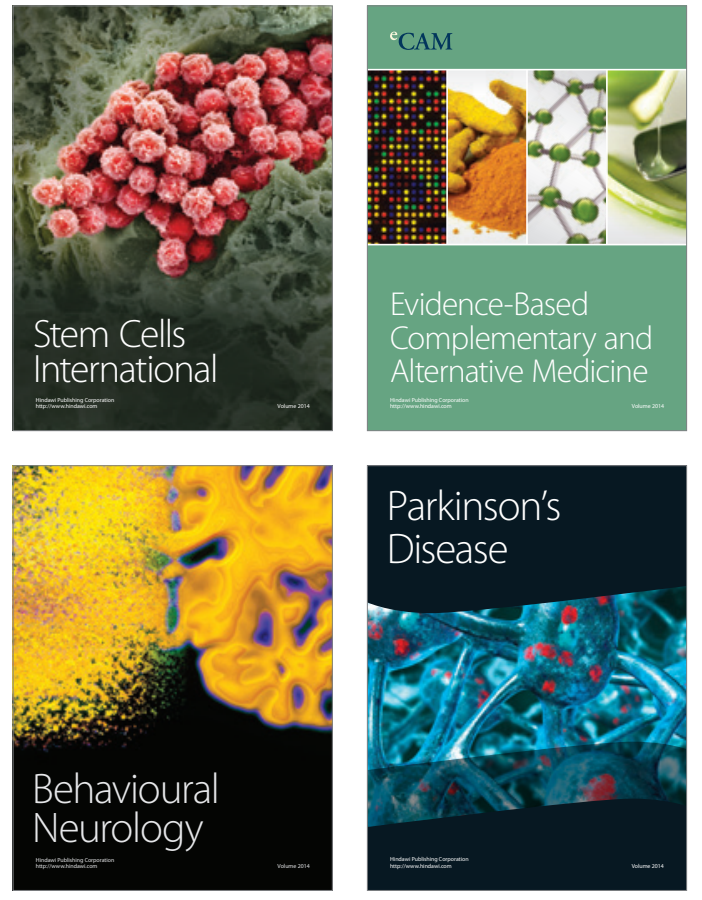
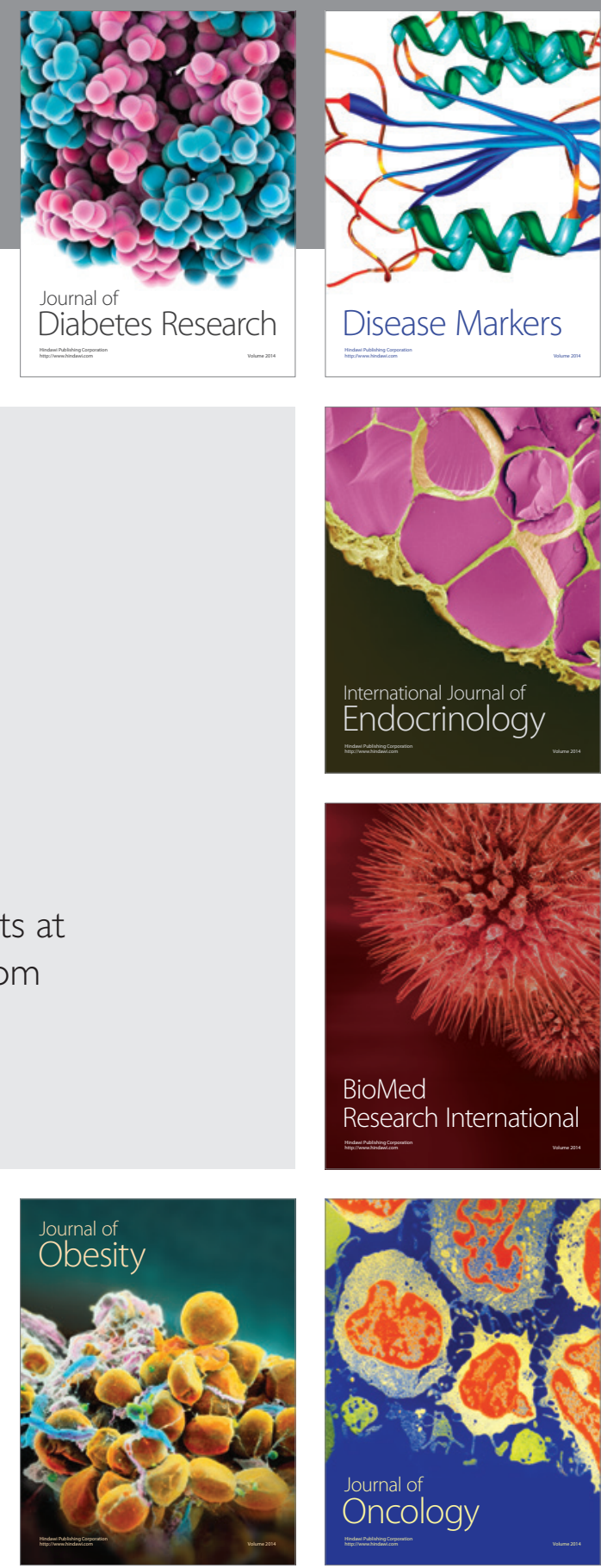

Disease Markers
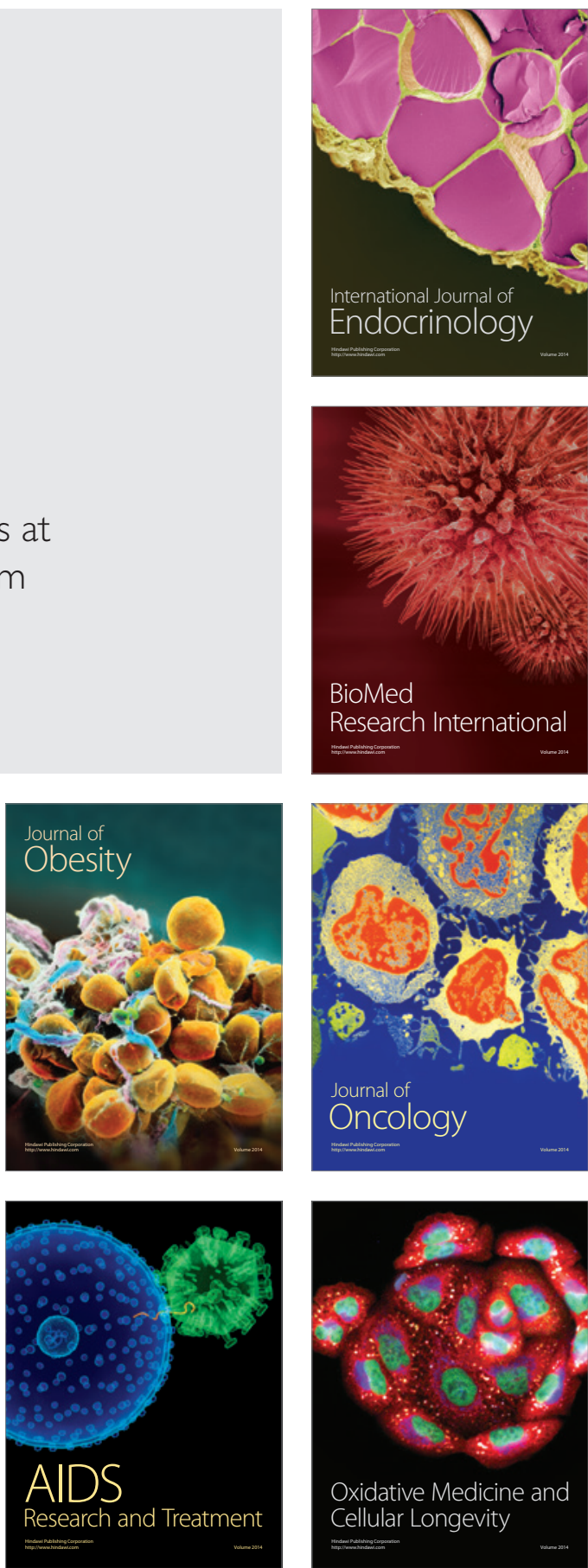